\title{
Slowed Temporal and Parietal Cerebrovascular Response in Patients with Alzheimer's Disease
}

\author{
Kenneth R. Holmes (iD, David Tang-Wai (i), Kevin Sam, Larissa McKetton, \\ Julien Poublanc, Adrian P. Crawley, Olivia Sobczyk, Melanie Cohn, James Duffin, \\ Maria Carmela Tartaglia, Sandra E. Black, Joseph A. Fisher, Bruce Wasserman, \\ David J. Mikulis
}

\begin{abstract}
Background: Recent investigations now suggest that cerebrovascular reactivity (CVR) is impaired in Alzheimer's disease $(\mathrm{AD})$ and may underpin part of the disease's neurovascular component. However, our understanding of the relationship between the magnitude of CVR, the speed of cerebrovascular response, and the progression of AD is still limited. This is especially true in patients with mild cognitive impairment (MCI), which is recognized as an intermediate stage between normal aging and dementia. The purpose of this study was to investigate $\mathrm{AD}$ and MCI patients by mapping repeatable and accurate measures of cerebrovascular function, namely the magnitude and speed of cerebrovascular response $(\tau)$ to a vasoactive stimulus in key predilection sites for vascular dysfunction in AD. Methods: Thirty-three subjects (age range: 52-83 years, 20 males) were prospectively recruited. CVR and $\tau$ were assessed using blood oxygen level-dependent MRI during a standardized carbon dioxide stimulus. Temporal and parietal cortical regions of interest (ROIs) were generated from anatomical images using the FreeSurfer image analysis suite. Results: Of 33 subjects recruited, 3 individuals were excluded, leaving 30 subjects for analysis, consisting of 6 individuals with early AD, 11 individuals with MCI, and 13 older healthy controls (HCs). $\tau$ was found to be significantly higher in the AD group compared to the HC group in both the temporal ( $p=0.03$ ) and parietal cortex $(p=0.01)$ following a one-way ANCOVA correcting for age and microangiopathy scoring and a Bonferroni post-hoc correction. Conclusion: The study findings suggest that AD is associated with a slowing of the cerebrovascular response in the temporal and parietal cortices.
\end{abstract}

RÉSUMÉ : Ralentissement de la réponse cérébrovasculaire dans le lobe temporal et le cortex pariétal chez des patients atteints de la maladie d'Alzheimer. Contexte : Des travaux de recherche récents suggèrent désormais que la réactivité cérébrovasculaire (RCV), en plus d'être altérée dans le cas de patients atteints de la maladie d'Alzheimer (MA), pourrait renforcer la composante neurovasculaire de cette maladie. Il n'empêche que notre compréhension des liens entre l'ampleur de la RCV, la rapidité d'une réponse cérébrovasculaire et la progression de la MA demeure limitée. Cela est particulièrement vrai dans le cas de patients aux prises avec des troubles cognitifs légers (TCL), lesquels sont reconnus comme étant des affections intermédiaires entre le vieillissement normal et la démence. L'objectif de cette étude est donc de se pencher sur les cas de patients atteints de MA et de TCL en établissant des mesures répétables et précises de la fonction cérébrovasculaire, à savoir l'ampleur et la rapidité de la réponse cérébrovasculaire $(\tau)$ à une stimulation vasoactive dans les principales régions de dysfonctionnement vasculaire associées à la MA. Méthodes : Au total, 33 sujets âgés de 52 à 83 ans, 20 étant des hommes, ont été recrutés de façon prospective. La RCV et la réponse cérébrovasculaire $(\tau)$ ont été évaluées au moyen d'IRM détectant le signal BOLD (blood oxygen level-dependent) à l'occasion d'une stimulation standardisée de dioxyde de carbone. Des résultats se rapportant à deux régions d'intérêt (lobe temporal et cortex pariétal) ont été obtenus à partir d'images anatomiques et du logiciel d'imagerie FreeSurfer. Résultats : Sur ces 33 sujets recrutés, 3 d'entre eux ont fini par être exclus. Sur ces 30 sujets, 6 étaient atteints d'une forme précoce de MA, 11 étaient aux prises avec des TCL alors que 13 autres ont intégré notre groupe de témoins en santé. À la suite d'une analyse correctrice de covariance à sens unique pour l'âge et pour des scores évaluant la sévérité de cas de microangiopathie et d'une correction de Bonferroni a posteriori, la réponse cérébrovasculaire $(\tau)$ s'est avérée sensiblement plus élevée dans le groupe de patients atteints de la MA en comparaison avec nos témoins en santé, et ce, qu'il s'agisse du lobe temporal $(\mathrm{p}=0,03)$ et du cortex pariétal $(\mathrm{p}=0,01)$.

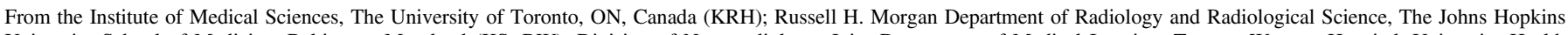

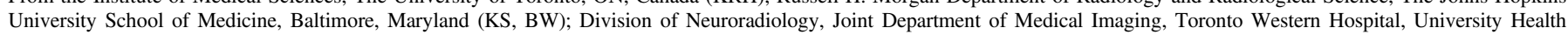

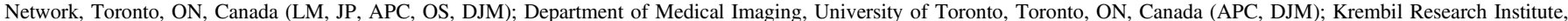

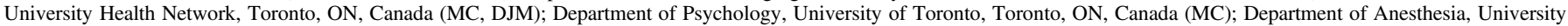

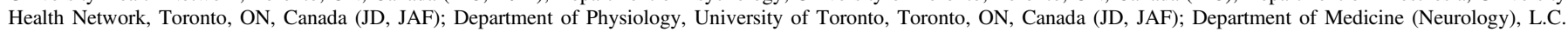

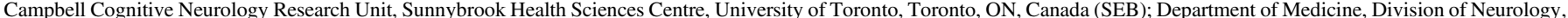

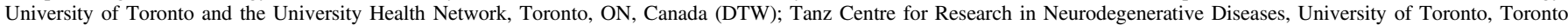
Western Hospital, University Health Network, Toronto, ON, Canada (MCT)

Received August 22, 2019. Final Revisions Submitted January 18, 2020. Date of Acceptance February 5, 2020.

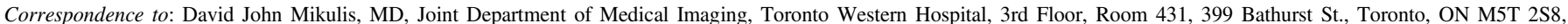
Email: david.mikulis@uhn.ca 
Conclusion : Les résultats de cette étude suggèrent en somme que la MA est associée à un ralentissement de la réponse cérébrovasculaire dans le lobe temporal et dans le cortex pariétal.

Keywords: Alzheimer's disease, Magnetic resonance imaging, Cerebrovascular reactivity, Cerebral blood flow regulation

doi:10.1017/cjn.2020.30

Can J Neurol Sci. 2020; 47: 366-373

\section{INTRODUCTION}

Alzheimer's disease (AD) is the most common contributing cause of dementia in the world, accounting for $60 \%-80 \%$ of the estimated 30-40 million cases worldwide. ${ }^{1,2}$ The presence of cerebrovascular disease (CVD) was originally used to demarcate $\mathrm{AD}$ and vascular dementia $(\mathrm{VaD})$; however, converging evidence now supports a major vascular component in AD. CVD and AD share numerous risk factors including obesity and hypertension during midlife, hypercholesterolemia, hyperhomocysteinemia, diabetes mellitus, and smoking. ${ }^{3-5}$ Additionally, atherosclerosis ratings in $\mathrm{AD}$ patients significantly correlate with neuritic plaque, neurofibrillary tangles, and cerebral amyloid angiopathy. 6,7

Cerebral hypoperfusion has been repeatedly observed in AD patients, with the temporal and parietal cortices found to be the most consistently affected. ${ }^{8-13}$ Moreover, reduced cerebral blood flow $(\mathrm{CBF})$ is demonstrable several years before the diagnosis of clinical $\mathrm{AD},{ }^{14}$ suggesting that vascular dysfunction may play an important role early in the disease.

Building on basal perfusion studies, there is now a growing body of evidence that cerebrovascular reactivity (CVR) in response to $\mathrm{CO}_{2}$ may be impaired in AD. ${ }^{15}$ Simply defined, CVR is the change in $\mathrm{CBF}$ in response to a given vasoactive stimulus that occurs during reductions in systemic blood pressure, hypercapnia, or during increases in neural activity. Initial studies using PET imaging techniques reported no differences in CVR between AD patients and elderly controls. ${ }^{16,17}$ However, early studies using alternative imaging modalities found CVR to be significantly impaired in AD. ${ }^{18,19}$ Since then, investigations using transcranial Doppler have more reliably demonstrated CVR impairment in AD patients. ${ }^{20,21}$ Most recently, studies utilizing blood oxygen level-dependent (BOLD) MRI and fixed inspired $\mathrm{CO}_{2}$ have found diminished CVR in both $\mathrm{AD}^{22,23}$ and mild cognitive impairment $(\mathrm{MCI})^{23}$, which is recognized as an intermediate stage between normal aging and dementia. These findings indicate that CVR deficits may occur early in the disease and could contribute to progression, though the spatial location of CVR deficits remains to be elucidated.

Beyond measuring CVR magnitude, assessing the speed of cerebrovascular response may also be of interest as it is an indicator of vascular compliance ${ }^{24}$ and may impact the important temporal linkage between neural activity and the resultant change in CBF. Richiardi and colleagues investigated the speed of cerebrovascular response to vasoactive stimuli in $\mathrm{AD}$ and MCI patients using BOLD MRI and a fixed inspired $\mathrm{CO}_{2}$ stimulus. ${ }^{25}$ They reported that both MCI and AD patients demonstrated a slowed vascular response globally when compared to healthy controls (HCs). However, further studies were needed to corroborate their findings.

Therefore, we applied an accurate, repeatable, and reproducible CVR methodology for measuring the magnitude and speed of the cerebrovascular response to $\mathrm{CO}_{2}$ in key predilection sites of $\mathrm{AD}$ in patients with MCI and early AD. The method uses BOLD MRI as a surrogate for CBF during precision control of end-tidal tensions of carbon dioxide $\left(\mathrm{P}_{\mathrm{ET}} \mathrm{CO}_{2}\right)$ and oxygen $\left(\mathrm{P}_{\mathrm{ET}} \mathrm{O}_{2}\right)$ thus providing a standardized and repeatable vasoactive stimulus. We hypothesized that reduced CVR and slowed vascular reactivity would occur in the temporal and parietal cortices of subjects with MCI and AD when compared to older HCs.

\section{Methods \\ Participants}

This study was approved by the Research Ethics Board at the University Hospital Network. Subjects were recruited through the memory clinic located at Toronto Western Hospital, where both written and informed consent were provided. Thirty-three subjects were recruited to participate in the study, consisting of 7 individuals with early AD, 12 individuals with MCI, and 14 older (HCs (age range: 52-83 years, 20 males). Diagnosis of MCI and AD patients was performed by a neurologist according to the 2011 guidelines set out by the National Institute on Aging and Alzheimer's Association consensus panels. ${ }^{26,27}$ Older $\mathrm{HC}$ was also recruited and screened according to the outlined inclusion and exclusion criteria. Subjects were asked to refrain from heavy exercise or caffeine intake on the day of the scan.

Inclusion criteria for this study were as follows: (i) a diagnosis of MCI (amnestic subtype) or early dementia due to AD; (ii) over 50 years of age; and (iii) deemed fit for investigation by the referring neurologist. Exclusion criteria for this study were as follows: (i) medical contraindications to controlled hypercapnia; (ii) incompatibility with 3-Tesla MRI; (iii) mixed $\mathrm{AD}$ and $\mathrm{VaD}$; (iv) history of stroke, transient ischemic attack (TIA), or hemodynamically significant carotid stenosis; (v) uncontrolled hypertension, dyslipidemia, or diabetes; (vi) history of unrelated neurological disease; (vii) pulmonary disease; and (viii) medications known to interfere with CVR measurements.

\section{Image Acquisition}

All studies were performed at the Toronto Western Hospital on a 3-Tesla GE MRI system (Signa HDx platform, GE Healthcare, Milwaukee, WI, USA) using an eight-channel phased array head coil. A third generation RespirAct ${ }^{\mathrm{TM}}$ gas blender was employed for control of $\mathrm{P}_{\mathrm{ET}} \mathrm{CO}_{2}$ during the BOLD CVR sequence. All patients underwent T1-weighted structural imaging, followed by a T2*-weighted axial single-shot BOLD CVR image, 2D FLAIR image, and a proton density/T2-weighted sequence. Full imaging parameters are included in Supplement 1.

\section{Vasodilatory Stimulus}

Assessing CVR requires the delivery of a vasoactive stimulus. In this study, a standardized hypercapnic breathing stimulus was delivered to each patient during BOLD MRI to fulfill this 

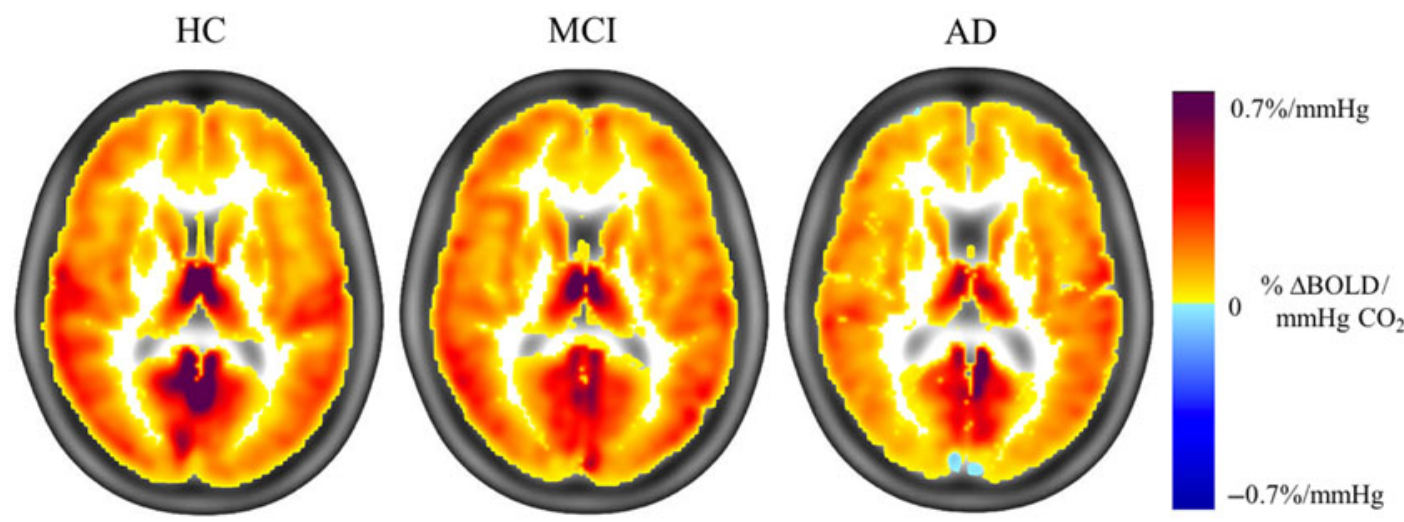

Figure 1: Maps of mean CVR in cortical GM in each group. Color-coded map of mean CVR in cortical GM for each group superimposed on a T1-weighted anatomical map in standard space. Purple/red/orange/yellow indicate a graded positive CVR response, while blues indicate a reduction in blood flow to the vasodilatory stimulus (steal physiology).
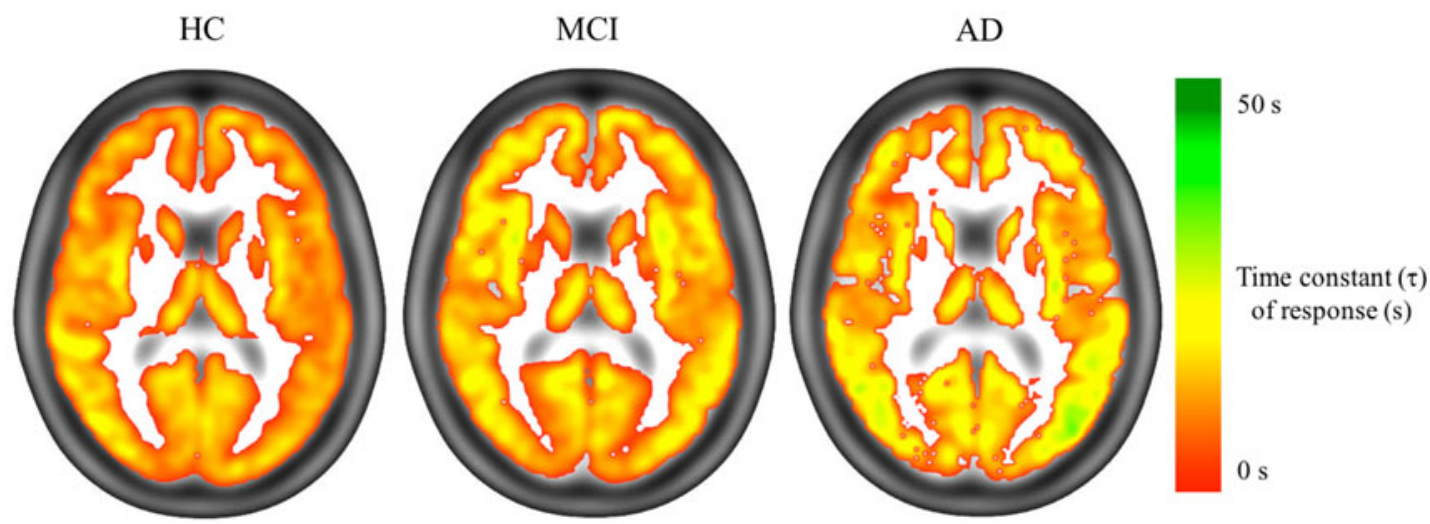

Figure 2: Maps of mean time constant $(\tau)$ of the cerebrovascular response in cortical GM in each group. Color-coded map of mean $\tau$ value in cortical GM for each group superimposed on a T1-weighted anatomical map in standard space. Reds and oranges indicate smaller $\tau$ values and a faster cerebrovascular response to hypercapnia, while greens indicate larger $\tau$ values and a slower cerebrovascular response.

requirement. Control of $\mathrm{P}_{\mathrm{ET}} \mathrm{CO}_{2}$ was achieved, enabling control of alveolar ventilation, using the RespirAct ${ }^{\mathrm{TM}}$ system (Thornhill Medical Inc., Toronto, Canada). For this study, a standardized $\mathrm{CO}_{2}$ inspiratory stimulus was implemented, consisting of the following: baseline for $120 \mathrm{~s}$ at subject's resting $\mathrm{P}_{\mathrm{ET}} \mathrm{CO}_{2}$, a hypercapnic step of $10 \mathrm{mmHg}$ above resting for $120 \mathrm{~s}$ (rise time $=$ two breaths), baseline for $150 \mathrm{~s}$, a hypocapnic step of $10 \mathrm{mmHg}$ below resting followed immediately by a gradual ramp up to $15 \mathrm{mmHg}$ above resting lasting $270 \mathrm{~s}$, and a final baseline for $120 \mathrm{~s}$, all during normoxia.

\section{Calculating CVR and Speed of Cerebrovascular Reactivity}

After initial processing of raw BOLD images, scans with greater than $2 \mathrm{~mm}$ of motion were discarded. CVR was then calculated as the regression coefficient of a linear, least-squares fit of the BOLD signal to the $\mathrm{P}_{\mathrm{ET}} \mathrm{CO}_{2}$ time series on a per voxel basis. ${ }^{28} \mathrm{CVR}$ is expressed as the percent change in BOLD signal per mmHg change in $\mathrm{P}_{\mathrm{ET}} \mathrm{CO}_{2}$. For visualization purposes, the CVR value in each voxel can be assigned a color and superimposed on the corresponding voxel of the anatomical images (using real-time linear interpolation) to produce a color-coded CVR map (Figure 1).
Calculating the speed of the cerebrovascular response has been previously described. ${ }^{24}$ In short, the BOLD response to the hypercapnic step change in $\mathrm{CO}_{2}$ was modeled by convolving the $\mathrm{P}_{\mathrm{ET}} \mathrm{CO}_{2}$ time series with a hemodynamic response function in the form of an exponential decay function, $\exp (-t / \tau)$ where $t$ is time and $\tau$ is the time constant of the cerebrovascular response. Pearson correlation testing was then used to identify the $\tau$ value most correlated with the observed BOLD response to the vasodilatory stimulus. This $\tau$ value represents the speed of response expressed in seconds. Therefore, smaller $\tau$ values reflect a faster cerebrovascular response, while larger $\tau$ values reflect a slower response. The $\tau$ value in each voxel is then assigned a color and superimposed on the corresponding voxel of the anatomical images (using real-time linear interpolation) to produce a color-coded $\tau$ map (Figure 2). Further details regarding BOLD signal processing and calculation of both CVR and speed of cerebrovascular response are included in Supplement 1.

\section{Partial Volume Correction}

Progressive cortical atrophy is a cardinal feature of AD and may cause a partial volume effect in imaging data. To address this potential confound, the analysis was confined to cortical gray 

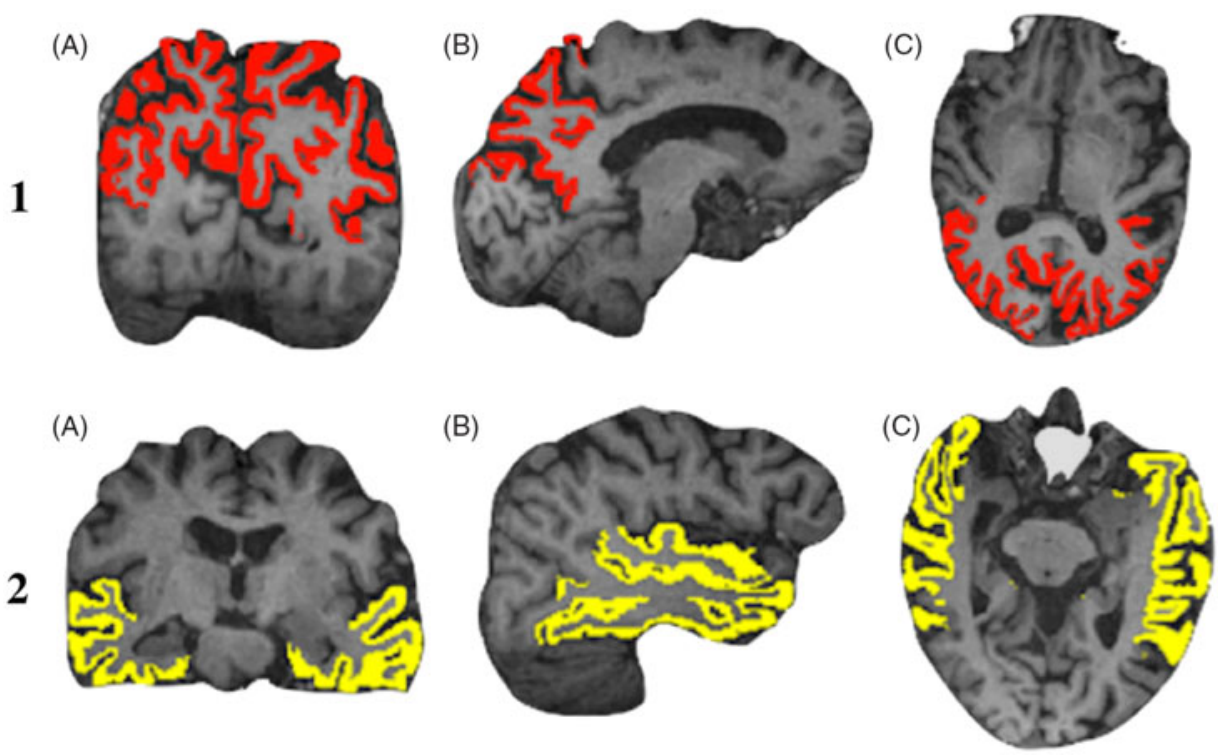

Figure 3: ROIs in an example subject. Panel 1 shows the (A) coronal, (B) sagittal, and (C) axial views of the parietal cortex ROI (red), whereas panel 2 shows the (A) coronal, (B) and sagittal, (C) axial views of the temporal cortex ROI (yellow).

matter (GM) and a correction was applied to both the CVR and $\tau$ values in each voxel to account for variability in the proportion of GM and WM within the voxel. This correction is further described in Supplement 1.

\section{Generation of ROIs}

Two ROIs were included in the analysis, namely the temporal and parietal cortices. These two regions were selected due to their established selective vulnerability in AD. The ROIs were generated from the T1-weighted anatomical images using the FreeSurfer image analysis suite. First, anatomical images underwent a bias field correction and GM/WM segmentation. Then, cortical surface models of the GM/WM boundary and pial surface were reconstructed, followed by parcellation and labeling of the cortical surface. Whole-lobe cortical ROIs were then constructed for the temporal and parietal cortex for each subject and visually inspected for quality (Figure 3).

\section{Assessment of White Matter Hyperintensity (WMH) Burden}

To ensure that any group differences in CVR or the speed of cerebrovascular response were not the result of group differences in small vessel disease, the WMH burden was scored for each patient as a surrogate measure of microvascular disease and added to subsequent statistical analyses as a covariate of no interest. To accomplish this, WMH burden was scored by an experienced neuroradiologist (DJM), blinded to group membership, for each patient according to the Fazekas scale using proton density/ T2-weighted images and 2D FLAIR images. ${ }^{29}$

\section{Statistical Analyses}

All statistical analyses were completed using IBM SPSS Statistics (IBM SPSS Statistics for Macintosh, Version 21.0, Armonk, NY, USA). To assess differences in CVR and speed of cerebrovascular response $(\tau)$ among $\mathrm{AD}, \mathrm{MCI}$, and $\mathrm{HC}$ groups, a one-way ANCOVA was carried out for both measures, CVR and $\tau$, in both ROIs, while correcting for age and Fazekas score. Also, the assumptions of homogeneity of variances and normality of residuals were tested using Levene's test and the Shapiro-Wilk test, respectively. In the event of a statistically significant finding, Bonferroni-corrected post-hoc testing assessed pairwise comparisons.

\section{ReSUlts}

Among the 33 subjects recruited to participate in this study, 3 individuals were excluded due to: (i) incomplete imaging protocol due to study drop-out $(n=1)$, (ii) poor/unsatisfactory acquisition quality $(n=1)$, and (iii) scanner-related image artifact $(n=1)$. Altogether, 30 subjects remained for analysis, consisting of $6 \mathrm{AD}$ subjects, $11 \mathrm{MCI}$, and 13 older HCs. Table 1 summarizes all demographic data for each group.

Descriptive statistics for CVR in each ROI in each group are summarized in Table 2 and visualized in Figure 4. Group maps of mean CVR in cortical GM are presented in Figure 1. The assumptions of homogeneity of variances and normality of residuals were found to be satisfactory. In the temporal cortex, mean CVR was greatest in the MCI group (measured as $\% \Delta \mathrm{BOLD} / \mathrm{mmHg}$ with standard deviations in Table 2) at 0.527 and lowest in the HC group at 0.515 , with $\mathrm{AD}$ possessing an intermediate mean CVR value of 0.519 . In the parietal cortex, mean CVR was greatest in the MCI group at 0.410 and lowest in the $\mathrm{AD}$ group at 0.365 , with $\mathrm{HC}$ possessing an intermediate mean CVR value of 0.384 . The between-groups ANCOVA found no significant main effect in either the temporal cortex, $F(2,29)=0.38, p=0.69$, or the parietal cortex, $F(2,29)=1.32, p=0.29$.

Descriptive statistics for speed of cerebrovascular response in each ROI in each group are summarized in Table 3 and visualized in Figure 5. Group maps of the time constant $(\tau)$ of the cerebrovascular response in cortical GM are presented in Figure 2. The assumptions of homogeneity of variances and normality of residuals were found to be satisfactory. In both the temporal and 


\section{Table 1: Patient demographics}

\begin{tabular}{l|l|c|c|c}
\hline \multirow{2}{*}{ Age } & & HC $(\boldsymbol{n}=\mathbf{1 3})$ & MCI $(\boldsymbol{n}=\mathbf{1 1})$ & AD $(\boldsymbol{n}=\mathbf{6})$ \\
\hline \multirow{3}{*}{ Gender } & Female & $63.9 \pm 10.2$ & $73.1 \pm 5.7$ & $70.5 \pm 5.8$ \\
\cline { 2 - 5 } & Male & 4 & 4 & 2 \\
\hline \multirow{4}{*}{$\begin{array}{l}\text { Vascular risk } \\
\text { actors }\end{array}$} & Hypertension & 1 & 7 & 4 \\
\cline { 2 - 5 } & Dyslipidemia & 1 & 2 & 2 \\
\cline { 2 - 5 } & Diabetes mellitus & 0 & 0 & 1 \\
\cline { 2 - 5 } & Smoking & 0 & 1 & 0 \\
\hline \multirow{4}{*}{ Medications } & AChE inhibitor & 0 & 0 & 1 \\
\cline { 2 - 5 } & Antidepressant & 1 & 1 & 2 \\
\cline { 2 - 5 } & Antihypertensive & 1 & 2 & 2 \\
\cline { 2 - 5 } & Statin & 1 & 1 & 1 \\
\cline { 2 - 5 } & Beta-blocker & 0 & 1 & 2 \\
\cline { 2 - 5 } & Platelet drug & 0 & 0 & 2 \\
\cline { 2 - 5 } & Insulin/Metformin & 0 & 0 & 1 \\
\hline \multirow{4}{*}{ Fazekas score } & & $0.69 \pm 0.75$ & $1.64 \pm 0.81$ & $1.00 \pm 0.63$ \\
\hline MoCA & & $\mathrm{n} / \mathrm{a}$ & $23.4 \pm 1.99$ & $18.3 \pm 1.71$ \\
\hline \multirow{2}{*}{ For MoCA } & & & & \\
\hline
\end{tabular}

For MoCA testing, $n=7$ for MCI and $n=4$ for AD.

Table 2: CVR in temporal and parietal ROIs across HC, MCI, and AD groups

\begin{tabular}{l|l|c|c|c}
\hline \multirow{2}{*}{ Cortical ROI } & Group & $\begin{array}{c}\text { Mean CVR } \\
(\% / \mathbf{m m H g})\end{array}$ & Std. Deviation & $\begin{array}{c}\text { Est. Marg. } \\
\text { Mean }\end{array}$ \\
\hline \multirow{2}{*}{ Temporal } & HC & 0.515 & 0.064 & 0.504 \\
\cline { 2 - 5 } & MCI & 0.527 & 0.136 & 0.544 \\
\cline { 2 - 5 } & AD & 0.519 & 0.094 & 0.512 \\
\hline \multirow{2}{*}{ Parietal } & HC & 0.384 & 0.060 & 0.365 \\
\cline { 2 - 5 } & MCI & 0.410 & 0.118 & 0.429 \\
\cline { 2 - 5 } & AD & 0.365 & 0.073 & 0.368 \\
\hline
\end{tabular}

parietal cortices, mean $\tau$ of the cerebrovascular response was greatest in the AD group at 44.5 and $48.3 \mathrm{~s}$, respectively. It was lowest in the HC group at 26.4 and $23.7 \mathrm{~s}$, respectively, with MCI possessing intermediate mean $\tau$ values of 34.2 and $34.3 \mathrm{~s}$, respectively. This indicates that the mean speed of cerebrovascular response was fastest in the HC group and slowest in the AD group in both ROIs. The between-groups ANCOVAs found significant effects in both the temporal and parietal cortices (temporal: $F(2,29)=3.93, p=0.03$; parietal: $F(2,29)=5.05$, $p=0.01)$. Bonferroni-corrected post-hoc testing revealed significantly increased $\tau$ in the AD group compared to the HC group in both the temporal $(p=0.03)$ and parietal cortex $(p=0.01)$; however, the MCI group was not significantly different from either group.

\section{Discussion}

Impaired cerebral vasoreactivity is now increasingly recognized as a potential contributor to $\mathrm{AD} .{ }^{30}$ This study aimed to evaluate both CVR and speed of cerebrovascular response to $\mathrm{CO}_{2}$ in amnestic MCI and AD patients using a repeatable and reproducible methodology. This was achieved with a gas blending apparatus that enabled tight control of arterial $\mathrm{pCO}_{2}$. This allowed delivery of a hypercapnic stimulus optimized for measuring both CVR and speed of cerebrovascular response. The initial abrupt $\mathrm{pCO}_{2}$ step change is ideal for assessing speed of response as gradual increases in $\mathrm{P}_{\mathrm{a}} \mathrm{CO}_{2}$ will make it difficult to resolve vascular responses with a time course shorter than that of the stimulus. On the other hand, the ramp component is delay insensitive, allowing for the CVR to be assessed with minimal confound due to delayed responses. In comparison, previous studies have generally used a fixed inspired $\mathrm{CO}_{2}$ challenge, which results in a biexponential rise in $\mathrm{P}_{\mathrm{a}} \mathrm{CO}_{2}$ that is influenced by minute ventilation and cannot be accurately quantified by measuring $\mathrm{P}_{\mathrm{ET}} \mathrm{CO}_{2} \cdot{ }^{31}$ Ultimately, we found that development of $\mathrm{AD}$ is associated with a slowing of the cerebrovascular response, and that this deficit cannot be explained by differences in age or microangiopathy. However, we were unable to detect any CVR impairment in either the MCI or AD groups. Nevertheless, our findings support the hypothesis that abnormal vascular responses may play a meaningful role in the pathophysiology of AD.

Failing to detect CVR impairment in either the temporal or parietal cortex was not entirely unexpected given the inconsistency of recent reports investigating CVR in AD, especially with respect to these two cortical regions. Our CVR findings are generally consistent with Yezhuvath and colleagues, who used BOLD MRI and fixed inspired $\mathrm{CO}_{2}$ to examine CVR in $17 \mathrm{AD}$ patients and 17 age-matched controls. ${ }^{22}$ Despite observing a resting CBF reduction in posterior cortical structures, the authors observed no significant CVR impairment in the temporal or parietal cortex. Conversely, our CVR results conflict with the findings of Cantin and colleagues, who reported a widespread CVR deficit in both $\mathrm{AD}(n=9)$ and MCI $(n=7)$ groups that was statistically significant in both the temporal and parietal cortices of both MCI and AD groups. ${ }^{23}$ Altogether, our findings refute the notion that CVR deficits occur in the temporal or parietal cortex in AD patients. This may be due to the study design that was employed that enabled precise control of the vasodilatory stimulus as well as the aggressive approach used to control partial volume averaging errors that can arise from brain atrophy. Nonetheless, CVR deficits may be present elsewhere in the brain. If that were the case, it would be unlikely that they are related to the characteristic hypoperfusion found in $\mathrm{AD}$, which typically predominates in the temporal and parietal cortices.

Our findings of slowed vasoreactivity in both the temporal and parietal cortex are consistent with the current body of work on the subject. Richiardi and colleagues reported a global slowing of the cerebrovascular response to inhaled $\mathrm{CO}_{2}$ in both $\mathrm{AD}$ and $\mathrm{MCI}$ patients when compared with older $\mathrm{HCs}^{25}$ in particular, a delayed response was found in the temporal and parietal cortices in the AD group. Cantin and colleagues also observed a different slope to the BOLD response to hypercapnia in MCI and AD groups compared to controls, suggesting that the temporal dynamics of CVR may be altered by the disease. ${ }^{23}$ Moreover, $\mathrm{AD}$ and MCI patients have been shown to elicit delayed BOLD responses to visual encoding tasks. ${ }^{32}$ An inference can be drawn that slowing of the vasodilatory vascular response may be due to a decrease in vascular compliance. Furthermore, it is tempting to link this loss of compliance with recent work in a rodent model of 
Table 3: Time constant $(\tau)$ of the cerebrovascular response to $\mathrm{CO}_{2}$ across $\mathrm{HC}, \mathrm{MCI}$, and $\mathrm{AD}$ groups

\begin{tabular}{l|l|c|c|c}
\hline Cortical ROI & \multicolumn{1}{c|}{ Group } & Mean $\tau(\mathbf{s})$ & Std. Deviation & Est. Marg. Mean (s) \\
\hline \multirow{3}{*}{ Temporal } & HC & 26.4 & 11.5 & 27.6 \\
\cline { 2 - 5 } & MCI & 34.2 & 12.9 & 32.7 \\
\cline { 2 - 5 } & AD & 44.5 & 7.8 & 44.6 \\
\hline \multirow{3}{*}{ Parietal } & HC & 23.7 & 12.9 & 25.1 \\
\cline { 2 - 5 } & MCI & 34.3 & 16.5 & 32.5 \\
\cline { 2 - 5 } & AD & 48.3 & 11.6 & 48.6 \\
\hline
\end{tabular}

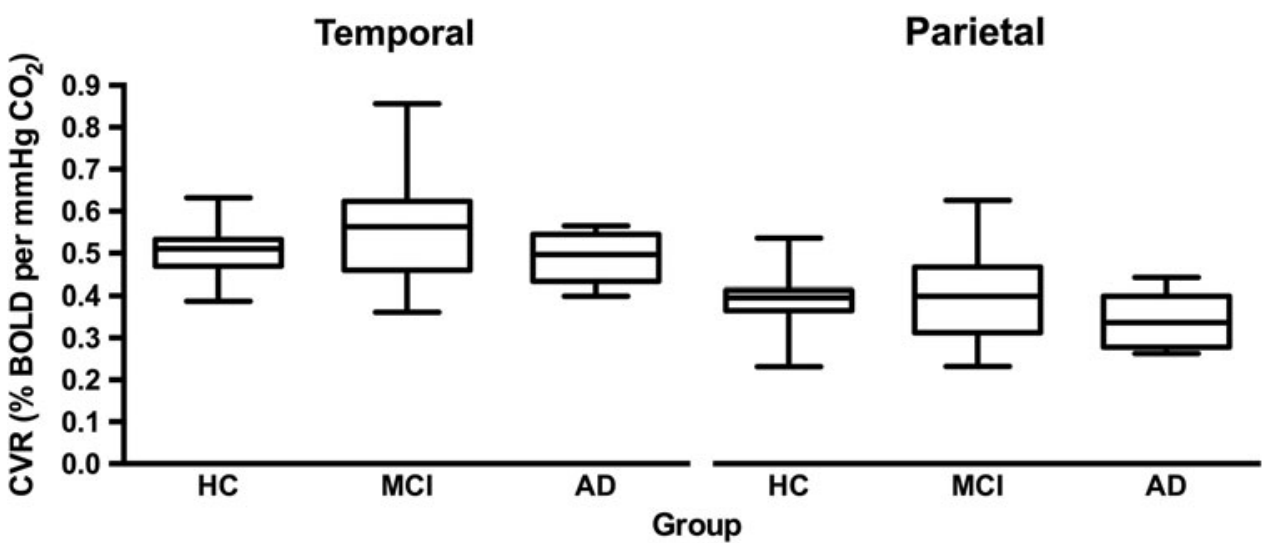

Figure 4: $C V R$ in the temporal and parietal cortex across groups. CVR values are reported as \% change in $B O L D$ per $\mathrm{mmHg}$ change on $\mathrm{P}_{E T} \mathrm{CO}_{2}$. Bars indicate minimum and maximum, boxes indicate the interquartile range, and the middle line denotes the median. No significant group differences were found in CVR in either cortical ROI.

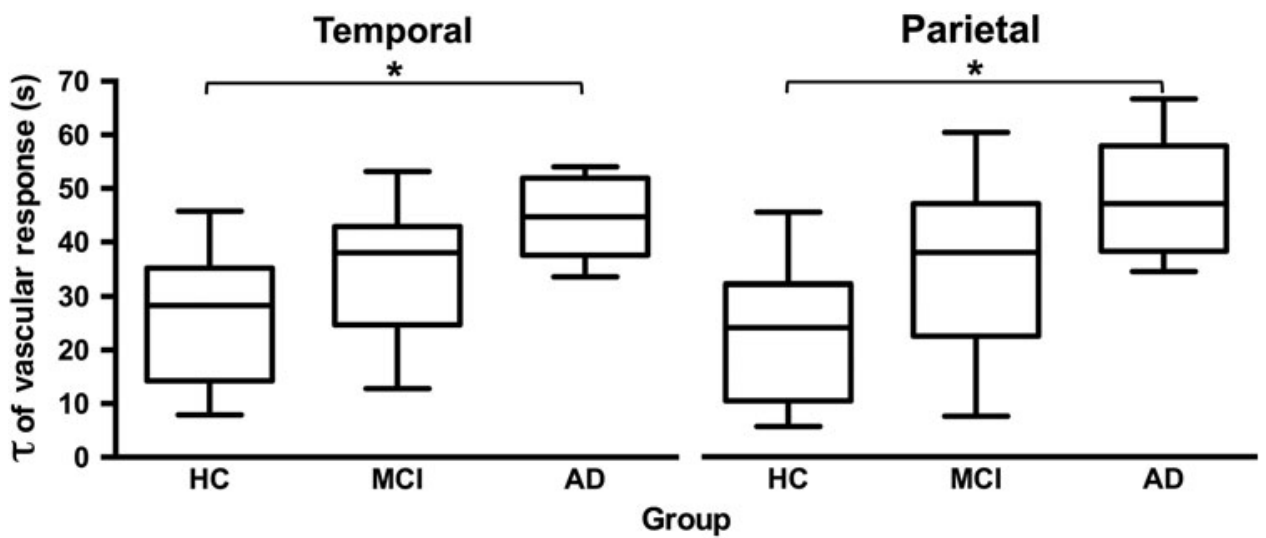

Figure 5: Time constant $(\tau)$ of the cerebrovascular response to $\mathrm{CO}_{2}$ in temporal and parietal cortex across groups. $\tau$ values are reported in seconds. Bars indicate minimum and maximum, boxes indicate the interquartile range, and the middle line denotes the median value. Higher values indicate a slower speed of response. * denotes significance compared to $H C(p<0.05)$. ANCOVA testing yielded a significant group effect on $\tau$ for both the temporal $(p=0.03)$ and parietal $(p=0.01)$ cortex, and Bonferroni-corrected post-hoc testing determined that $\tau$ was significantly greater in the $\mathrm{AD}$ group than the $\mathrm{HC}$ group in both cortical ROIs $(p=0.03$ and 0.01 , respectively).

AD showing radial bands of amyloid protein deposition separating endothelial cells from glial foot processes in the cerebral microvessels. ${ }^{33}$

A slowed vascular response may directly confer an ischemic burden present in AD by adding a significant delay between neural activity and the resultant increase in CBF to support it.
While the associated vascular deficit would be temporary, it may give rise to short ischemic 'events' during neuronal signaling activity. Due to the protracted disease latency of $\mathrm{AD}$, the relatively small burden of these events may gradually accumulate over years or even decades, contributing to the total ischemic burden in $\mathrm{AD}$ in a meaningful way. In addition, as an indicator of 
poor vascular compliance, reduced speed of response in cerebral vessels may partially explain findings of impaired cerebral autoregulation in $\mathrm{AD} .{ }^{34}$ Lastly, reduced speed of the cerebrovascular response may also reflect a mechanism for impaired glymphatic flow in AD. A recent study by Peng and colleagues found that in a mouse model of AD, glymphatic transport was significantly reduced compared to wild-type. ${ }^{35}$ The driving force of glymphatic transport is still not fully understood, but recent evidence indicates that smooth muscle pulsation may drive CSF into the interstitial compartment. ${ }^{36,37}$ Slowed vasoreactivity may indicate impaired arteriolar pulsatility and, potentially, poor clearance of amyloid- $\beta$ and other waste products. Together, these represent three plausible mechanisms by which reduced speed of the cerebrovascular response could contribute to the vascular component of $\mathrm{AD}$.

\section{Limitations}

The main limitation of this study relates to sample size. Given the modest sample, our study may be underpowered to detect alterations in CVR. Also, this sample does suffer somewhat from a discrepancy in ages and prevalence of vascular risk factors between the groups. Theoretically, age may alter CVR or speed of vascular response. Additionally, vascular risk factors may reflect a greater burden of cerebrovascular disease, which can impair vascular responses. These potential confounders were mitigated by adding age and Fazekas score as a surrogate for cerebral microvascular disease to the statistical analyses as covariates of no interest.

The use of BOLD MRI as a surrogate measure of CBF may represent another potential limitation of this study as BOLD MRI does not directly measure changes in CBF. Instead, BOLD is sensitive to changes in the voxel concentration of deoxyhemoglobin $(\mathrm{dHb})$. Changes in $\mathrm{CBF}$ will influence $\mathrm{dHb}$ concentration; however, changes in the cerebral metabolic rate of $\mathrm{O}_{2}\left(\mathrm{CMRO}_{2}\right)$ and cerebral blood volume can also impact $\mathrm{dHb}$ concentration and, by extension, may create a nonlinearity effect in the BOLD signal or create a between-subject CVR scaling effect due to variable concentrations of $\mathrm{dHb}$ at rest. However, it is thought that $\mathrm{CMRO}_{2}$ is unaffected and changes in blood volume are negligible during a hypercapnic stimulus, though this cannot be definitively ruled out in this study.

\section{Conclusion}

Our findings indicate that AD is associated with a slowing of the cerebrovascular response in the temporal and parietal cortex. This preliminary work may provide new insights into the role of vascular dysregulation in the pathogenesis and progression of AD. Further evaluation of the relationship between speed of the cerebrovascular response and relevant cognitive measures may identify specific contributions of hemodynamic impairment to the functional progression of $\mathrm{AD}$, and thus may be of interest for future studies. Finally, early detection of future AD may be possible at the MCI stage of the disease by comparing the speed of response of MCI subjects against normal control atlases using an as yet to be determined threshold defined by the number of standard deviations below normal responses. If this could be validated in a larger sample size, it could represent a valuable clinical predictor of AD. With disease-modifying therapies on the horizon, this may prove clinically useful in identifying candidates for therapy.

\section{ACKNOWLEDGMENTS}

We are grateful to the Toronto Western Hospital, including research coordinator Abby Skanda, and MR imaging technologists Keith Ta and Eugen Hlasny.

\section{Statement OF AUTHORSHIP}

$\mathrm{KRH}$ was involved in all aspects of the research including research design, acquisition of data, image processing, statistical analysis, and manuscript generation. DTW and MCT assessed and selected subjects for study inclusion. KS, LM, OS, JD, JAF, $\mathrm{JP}$, and APC assisted in implementation of breathing protocol, image acquisition and post-processing methodology. MC provided neuropsychological protocol, data collection, and analysis. $\mathrm{BW}$ was involved in development of external funding proposal and advised on implementation of research protocol. SEB and DJM were members of KRH's MSc program advisory committee and supervised all aspects of this research. All authors read and approved the final manuscript.

\section{CONFLict of InTEREST}

KRH and DM received financial support for this work from the Canadian Institutes of Health Research. DM received a grant from the American Society of Neuroradiology during the conduct of this study. JF and JD are senior scientists at Thornhill Research Inc. (TRI), a spin-off company affiliated with the University Health Network that developed the RespirAct ${ }^{\mathrm{TM}}$, a non-commercial research tool assembled by TRI to enable cerebrovascular reactivity studies. JF and DM hold a patent related to the RespirAct ${ }^{\mathrm{TM}}$. Also, DM is a shareholder in TRI and reports non-financial support from TRI. OS is a part time employee of TRI. Outside of the submitted work, SEB reports institutional grants from GE Healthcare, Eli Lilly, Biogen, Novartis, Genentech, Optina, Roche, and personal fees from Novartis, Roche, and Eli Lilly. The remaining authors declare that the research was conducted in the absence of any commercial or financial relationships that could be construed as a potential conflict of interest.

\section{SUPPLEMENTARY MATERIAL}

To view supplementary material for this article, please visit https://doi.org/10.1017/cjn.2020.30.

\section{REFERENCES}

1. Barker WW, Luis CA, Kashuba A, et al. Relative frequencies of Alzheimer disease, Lewy body, vascular and frontotemporal dementia, and hippocampal sclerosis in the State of Florida Brain Bank. Alzheimer Dis Assoc Disord. 2002;16(4): 203-212.

2. Prince M, Bryce R, Albanese E, Wimo A, Ribeiro W, Ferri CP. The global prevalence of dementia: a systematic review and metaanalysis. Alzheimer's Dement. 2013;9(1):63-75.

3. Gorelick PB, Scuteri A, Black SE, et al. Vascular contributions to cognitive impairment and dementia: a statement for healthcare professionals from the American Heart Association/American Stroke Association. Stroke. 2011;42(9):2672-2713.

4. Meng XF, Yu JT, Wang HF, et al. Midlife vascular risk factors and the risk of Alzheimer's disease: a systematic review and metaanalysis. J Alzheimer's Dis. 2014;42(4):1295-1310.

5. Tolppanen AM, Ngandu T, Kåreholt I, et al. Midlife and late-life body mass index and late-life dementia: results from a prospective population-based cohort. J Alzheimer's Dis. 2014;38(1): 201-209. 
6. Beach TG, Wilson JR, Sue LI, et al. Circle of Willis atherosclerosis: association with Alzheimer's disease, neuritic plaques and neurofibrillary tangles. Acta Neuropathol. 2007;113(1):13-21.

7. Yarchoan M, Xie SX, Kling MA, et al. Cerebrovascular atherosclerosis correlates with Alzheimer pathology in neurodegenerative dementias. Brain. 2012;135(12):3749-3756.

8. Eberling JL, Jagust WJ, Reed BR, Baker MG. Reduced temporal lobe blood flow in Alzheimer's disease. Neurobiol Aging. 1992; 13(4):483-491.

9. Farkas E, Luiten PG. Cerebral microvascular pathology in aging and Alzheimer's disease. Prog Neurobiol. 2001;64(6): $575-611$.

10. Imran MB, Kawashima R, Awata S, et al. Tc-99m HMPAO SPECT in the evaluation of Alzheimer's disease: correlation between neuropsychiatric evaluation and CBF images. J Neurol Neurosurg Psychiatry. 1999;66(2):228-232.

11. Johnson NA, Jahng GH, Weiner MW, et al. Pattern of cerebral hypoperfusion in Alzheimer disease and mild cognitive impairment measured with arterial spin-labeling MR imaging: initial experience. Radiology. 2005;234(3):851-859.

12. Du AT, Jahng GH, Hayasaka S, et al. Hypoperfusion in frontotemporal dementia and Alzheimer disease by arterial spin labeling MRI. Neurology. 2006;67(7):1215-1220.

13. Verclytte $\mathrm{S}$, Lopes R, Lenfant $\mathrm{P}$, et al. Cerebral hypoperfusion and hypometabolism detected by arterial spin labeling MRI and FDGPET in early-onset Alzheimer's disease. J Neuroimaging. 2016; 26(2):207-212.

14. Hays CC, Zlatar ZZ, Wierenga CE. The utility of cerebral blood flow as a biomarker of preclinical Alzheimer's disease. Cell Mol Neurobiol. 2016;36(2):167-179.

15. Glodzik L, Randall C, Rusinek H, De Leon MJ. Cerebrovascular reactivity to carbon dioxide in Alzheimer's disease. J Alzheimer's Dis. 2013;35(3):427-440.

16. Jagust WJ, Eberling JL, Reed BR, Mathis CA, Budinger TF. Clinical studies of cerebral blood flow in Alzheimer's disease. Ann N Y Acad Sci. 1997;826:254-262.

17. Kuwabara Y, Ichiya Y, Otsuka M, Masuda K, Ichimiya A, Fujishima M. Cerebrovascular responsiveness to hypercapnia in Alzheimer's dementia and vascular dementia of the Binswanger type. Stroke. 1992;23(4):594-598.

18. Oishi M, Mochizuki Y, Takasu T. Regional differences in cerebrovascular reactivity to acetazolamide in Alzheimer's disease. J Clin Neurosci. 1999;6(5):380-381.

19. Stoppe G, Schütze R, Kögler A, et al. Cerebrovascular reactivity to acetazolamide in (senile) dementia of Alzheimer's type: relationship to disease severity. Dementia. 1995;6(2):73-82.

20. Bär KJ, Boettger MK, Seidler N, Mentzel HJ, Terborg C, Sauer H. Influence of galantamine on vasomotor reactivity in alzheimer's disease and vascular dementia due to cerebral microangiopathy. Stroke. 2007;38(12):3186-3192.

21. Sabayan B, Jansen S, Oleksik AM, et al. Cerebrovascular hemodynamics in Alzheimer' $s$ disease and vascular dementia : a metaanalysis of transcranial Doppler studies. Ageing Res Rev. 2012; 11(2):271-277.
22. Yezhuvath US, Uh J, Cheng Y, et al. Forebrain-dominant deficit in cerebrovascular reactivity in Alzheimer's disease. Neurobiol Aging. 2012;33(1):75-82.

23. Cantin S, Villien M, Moreaud O, et al. Impaired cerebral vasoreactivity to $\mathrm{CO} 2$ in Alzheimer's disease using BOLD fMRI. Neuroimage. 2011;58(2):579-587.

24. Poublanc J, Crawley AP, Sobczyk O, et al. Measuring cerebrovascular reactivity: the dynamic response to a step hypercapnic stimulus. J Cereb Blood Flow Metab. 2015;35(11):1746-1756.

25. Richiardi J, Monsch AU, Haas T, et al. Altered cerebrovascular reactivity velocity in mild cognitive impairment and Alzheimer's disease. Neurobiol Aging. 2015;36(1):33-41.

26. Albert MS, DeKosky ST, Dickson D, et al. The diagnosis of mild cognitive impairment due to Alzheimer's disease: recommendations from the National Institute on Aging-Alzheimer's Association workgroups on diagnostic guidelines for Alzheimer's disease. Alzheimer's Dement. 2011;7(3):270-279.

27. McKhann GM, Knopman DS, Chertkow H, et al. The diagnosis of dementia due to Alzheimer's disease: recommendations from the National Institute on Aging-Alzheimer's Association workgroups on diagnostic guidelines for Alzheimer's disease. Alzheimer's Dement. 2011;7(3):263-269.

28. Fierstra J, Poublanc J, Han JS, et al. Steal physiology is spatially associated with cortical thinning. J Neurol Neurosurg Psychiatry. 2010;81(3):290-293.

29. Fazekas F, Chawluk JB, Alavi A. MR signal abnormalities at 1.5 Tin Alzheimer's dementia and normal aging. Am J Neuroradiol. 1987;8(3):421-426.

30. Kisler K, Nelson AR, Montagne A, Zlokovic B V. Cerebral blood flow regulation and neurovascular dysfunction in Alzheimer disease. Nat Rev Neurosci. 2017;18(7):419-434.

31. Fisher JA. The $\mathrm{CO} 2$ stimulus for cerebrovascular reactivity: fixing inspired concentrations vs. targeting end-tidal partial pressures. J Cereb Blood Flow Metab. 2016;36(6):1004-1011.

32. Rombouts SA, Goekoop R, Stam CJ, Barkhof F, Scheltens P. Delayed rather than decreased BOLD response as a marker for early Alzheimer's disease. Neuroimage. 2005;26(4):10781085.

33. Kimbrough IF, Robel S, Roberson ED, Sontheimer H. Vascular amyloidosis impairs the gliovascular unit in a mouse model of Alzheimer's disease. Brain. 2015;138(12):3716-3733.

34. den Abeelen AS, Lagro J, van Beek AH, Claassen JA. Impaired cerebral autoregulation and vasomotor reactivity in sporadic Alzheimer's disease. Curr Alzheimer Res. 2014;11(1):11-17.

35. Peng W, Achariyar TM, Li B, et al. Suppression of glymphatic fluid transport in a mouse model of Alzheimer's disease. Neurobiol Dis. 2016;93:215-225.

36. Iliff JJ, Wang M, Liao Y, et al. A paravascular pathway facilitates CSF flow through the brain parenchyma and the clearance of interstitial solutes, including amyloid $\beta$. Sci Transl Med. 2012; 4(147):147ra111.

37. Iliff JJ, Wang M, Zeppenfeld DM, et al. Cerebral arterial pulsation drives paravascular CSF-Interstitial fluid exchange in the murine brain. J Neurosci. 2013;33(46):18190-18199. 